\title{
Root reconstruction for proximal repair in acute type $A$ aortic dissection
}

\author{
Yunxing Xue ${ }^{1,2,3 \#}$, Qing Zhou ${ }^{2,3 \#}$, Jun Pan ${ }^{2,3}$, Hailong Cao ${ }^{1,2,3}$, Fudong Fan ${ }^{2,3}$, Xiyu Zhu ${ }^{2,3}$, Hoshun Chong ${ }^{2,3}$, \\ Dongjin Wang ${ }^{1,2,3}$
}

${ }^{1}$ Nanjing Drum Tower Hospital Clinical College of Nanjing Medical University, Nanjing 210008, China; ${ }^{2}$ Department of Cardiothoracic Surgery, Nanjing Drum Tower Hospital, The Affiliated Hospital of Nanjing University Medical School, Nanjing 210008, China; ${ }^{3}$ Institute of Cardiothoracic Vascular Disease, Nanjing University, Nanjing 210008, China

Contributions: (I) Conception and design: D Wang, Y Xue, Q Zhou; (II) Administrative support: D Wang; (III) Provision of study materials or patients: Q Zhou, J Pan, H Cao, F Fan; (IV) Collection and assembly of data: Y Xue, X Zhu, H Chong; (V) Data analysis and interpretation: Y Xue; (VI) Manuscript writing: All authors; (VII) Final approval of manuscript: All authors.

\#These authors contributed equally to this work.

Correspondence to: Dongjin Wang, MD, PhD. The Affiliated Drum Tower Hospital of Nanjing University Medical School, 321 Zhongshan Road, Nanjing 210008, China. Email: dongjinwang_gl@163.com.

Background: Retrospective compared the results of root reconstruction and root replacement for acute type A aortic dissection ( ATAAD) patients and observed the rate of aortic insufficiency (AI) and aortic root dilation in the midterm follow-up period.

Methods: From 2008-2016, 427 ATAAD patients received surgical therapy in our center. There were 328 male and 99 female patients, aging from 22 to 83 years with a mean age of $(51.1 \pm 12.5)$ years. These patients were divided into two major groups: 298 cases with root reinforcement reconstruction (Root Reconstruction), 129 cases with Bentall procedure (Root Replacement).

Results: The 30-day mortality was 7.7\% (33/427), while no difference between the 2 procedures $(8.1 \%$ and $7.0 \%, \mathrm{P}=0.844)$. Cross-clamp, cardiopulmonary bypass, and circulatory arrest times of all the patients were $252.5 \pm 78.1,173.6 \pm 68.9,30.7 \pm 9.5$ minutes, respectively. In the average follow-up time of $(34.5 \pm 26.1)$ months, midterm survival rates were similar between the 2 procedures $(86.2 \%$ and $86.0 \%, \mathrm{P}=0.957)$. Only one patient received redo Bentall procedure because of severe aortic regurgitation and dilated aortic root $(50 \mathrm{~mm})$ in the Root Reconstruction Group.

Conclusions: The indication of root management of ATAAD is based on the diameter of aortic root, structure of aortic root, and the dissection involvement. For most ATAAD patients, aortic root reinforcement reconstruction is a feasible and safe method.

Keywords: Aortic dissection; root repair; Bentall

Submitted Jul 18, 2019. Accepted for publication Oct 08, 2019.

doi: $10.21037 /$ jtd.2019.10.62

View this article at: http://dx.doi.org/10.21037/jtd.2019.10.62

\section{Introduction}

Acute type A aortic dissection (ATAAD) is still one of the severe lethal disease even with increasing rate of surgical therapy and treatment success. More complexity the surgical treatment and more risk of bleeding related to surgery, higher mortality and morbidity will be postoperative. The major factors influencing the complexity of the procedure are root and arch management. We discuss the safety and reliable of root preservation technique based on our center's experience.

The proximal involvement of ATTAD will induce aortic insufficiency (AI). The main etiology of AI is the dilation of aortic sinus induced by dissection, while the aortic valves are structurally complete in most cases. Supra-coronary 
replacement of ascending aorta is technical easy and can accept low mortality, but the non-supporting structure of aortic root will develop obvious AI and aortic root dilation. A more aggressive surgical strategy involves excision of the dissected root and replacement with a composite valve-graft conduit (Bentall procedure). Bentall procedure can avoid reoperation, but the risk of valve-associated endocarditis and the burden of anti-coagulation and structural valve degradation still exist. Recently, valve sparing root replacement (VSRR) are used by some experienced surgeon in high-volume centers. The follow-up results revealed that the perioperative mortality is acceptable and reoperation rate is low. But complex surgical manipulation and the injury risk of coronary artery lead VSRR more limited.

We improved the conservative reconstruction method using Dacron felts, and reshaped the proximal root through planting a ring Dacron felt inside the native vessel. The four-layers structure created by neointima, native intima, neomedia and native adventitia is physiological and easy to surgical hemostasis. We conclude the experience and analyze the complication for AI and root dilation in this retrospective study.

\section{Methods}

\section{Patients}

From January 2008 through December 2016, 427 ATAAD patients (328 male and 99 female) received surgical therapy in our center. All patients were diagnosed by computerized tomography angiogram (CTA) and underwent surgical therapy during 2 weeks after disease sudden-onset. The current study was approved by the institutional review board of Nanjing Drum Tower Hospital (No. BL2014004). The requirement to obtain informed consent from the patient was waived, and all authors had full control of the data and information in this study.

\section{Indications}

After the patient checked by CTA and TTE, we got the data of the location of intimal entry, the diameter of aortic sinus, the degree of AI, the involvement of dissection and the malperfusion situation of important organs. For root management, we choose Bentall procedure for patients with the intimal entry in the sinus, aortic sinus dilation $(\geq 45 \mathrm{~mm})$, diseased valve with moderate or greater AI $(\geq 2+)$ or severely involvement of coronary artery ostia. Root reinforcement reconstruction method is adopted in the patients with the diameter of aortic sinus $<45 \mathrm{~mm}$, AI without diseased valve, intimal entry out of sinus and without severely involvement coronary artery ostia. If the patency and integrity of the coronary ostia is damaged, coronary artery bypass grafting (CABG) is necessary. We divide the 427 cases into two groups: 298 cases with root reinforcement reconstruction (Root Reconstruction Group), and 129 cases with Bentall procedure (Root Replacement Group).

\section{Surgical approach}

All patients underwent intravenous and inhaled anesthesia, endotracheal intubation, upper and lower extremity arterial puncture monitoring blood pressure and placing an esophageal ultrasound probe. After exposure the right axillary artery and right femoral artery, we open the chest in the middle line and exposure the supra-arch vessels. After systemic heparinization, we constructed the cardiopulmonary bypass through right axillary artery and right femoral artery into arteries and cavity drainage into right atrium. Routine retrograde myocardial perfusion was used through coronary sinus. When the bladder temperature (core temperature) dropped to $18-22{ }^{\circ} \mathrm{C}$, we arrested the systemic circulation and perfused the cerebral with selected cerebral perfusion (SCP) from right axillary artery. The distal repair method according to different situations. Generally, in the patients with arch dilation $(\geq 45 \mathrm{~mm})$ and intima tears in the arch and damaged structure of arch, we replaced the total arch using artificial blood vessels with four bifurcations. Frozen elephant trunk was applied the same time if replacing the total arch. Otherwise, partial aortic arch replacement or antegradeimplantation arch stent were used (two kinds of stents were introduced in our center previously) $(1,2)$. Core temperature begun to recover after anastomosis finished. During the rewarm phase, we repaired the aortic root. The root reinforcement reconstruction method is as follows: we wiped out all thrombus in the dissected aortic root and placed proper shape Dacron patch between the outer membrane and inner membrane to act as the new middle layer of the root, and Dacron felt was placed inside the inner membrane, after that the new four-layers root was sutured with continuous stitches. Then the reconstructed root was anastomosed with artificial vessel (Figure 1). Bentall procedure was implanted as normal. Great saphenous vein was used for CABG cases. We had done concomitant procedures based on situations. Details are list in Table 1 . 

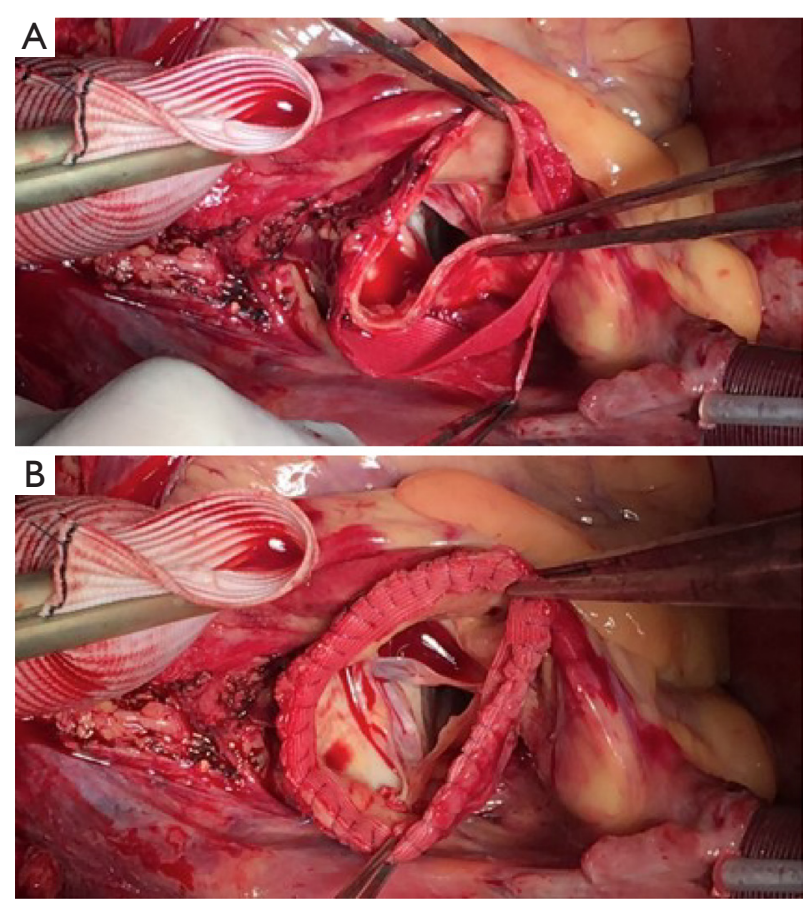

Figure 1 Image illustration of intra-operative view. Dacron felts is inserted between the outer and inner layer of aorta (A). The fourlayer neo-root is sutured with continuous stitches (B).

\section{Follow-up}

All patients received CTA and TTE in the following 1, 6 and 12 months and annually after surgery to complete our follow-up. We measured diameter at the level of the aortic sinus and the degree of AI. If the degree of AI $\geq 2$ or the diameter of aortic sinus $\geq 45 \mathrm{~mm}$, we defined it as new aortic root events.

\section{Statistical analysis}

Continuous variables are stated as mean \pm standard deviation. Categorical variables are stated as absolute numbers and proportions. Data analysis was performed using SPSS 22 Statistics software (IBM, Herrenberg, Germany). Kaplan-Meier analysis was used for evaluation of survival. Differences in categorical variables were analyzed using the $\chi^{2}$ test. Differences in continuous variables were tested using $t$-test, or Mann-Whitney $\mathrm{U}$-test, respectively. Multivariate analysis was performed using a binary logistic regression model to discriminate independent risk factors for 30-day mortality. We stated the odds ratio (OR) and the $95 \%$ confidence interval $(\mathrm{CI})$. A value of $\mathrm{P}<0.05$ was

Table 1 Indications for different root therapy method

\begin{tabular}{|c|c|c|c|c|}
\hline Categories & Whole group $(n=427)$ & Root Reconstruction ( $\mathrm{n}=298)$ & Root Replacement ( $n=129)$ & $P$ valve \\
\hline Aortic regurgitation (0-4), N (\%) & & & & $<0.001$ \\
\hline 0 & $61(14.3)$ & $61(20.5)$ & 0 & \\
\hline 1 & $127(29.7)$ & $124(41.6)$ & $3(2.3)$ & \\
\hline 1.5 & $67(15.7)$ & $59(19.8)$ & $8(6.2)$ & \\
\hline 2 & $81(19.0)$ & $38(12.8)$ & $43(33.3)$ & \\
\hline 2.5 & $1(0.2)$ & $1(0.3)$ & 0 & \\
\hline 3 & $59(13.8)$ & $11(3.7)$ & $48(37.2)$ & \\
\hline 4 & $33(7.7)$ & $5(1.7)$ & $28(21.7)$ & \\
\hline Diameter of aortic sinus (mm) & $41.4 \pm 7.3$ & $38.4 \pm 5.3$ & $48.6 \pm 6.2$ & $<0.001$ \\
\hline Coronary artery dissection involve & & & & 0.001 \\
\hline Neri A (number) & 30 & 22 & 8 & \\
\hline Neri B (number) & 16 & 5 & 11 & \\
\hline Neri C (number) & 4 & 0 & 4 & \\
\hline
\end{tabular}


Table 2 Clinical data

\begin{tabular}{|c|c|c|c|c|}
\hline Categories & Whole group $(n=427)$ & Root Reconstruction ( $n=298$ ) & Root Replacement ( $n=129)$ & $P$ valve \\
\hline \multicolumn{5}{|l|}{ Age (years) } \\
\hline \multicolumn{5}{|l|}{ Gender, N (\%) } \\
\hline Male & $328(76.8)$ & $222(74.5)$ & $106(82.2)$ & 0.084 \\
\hline Hypertension, N (\%) & $298(69.8)$ & $242(81.2)$ & $56(43.4)$ & $<0.001$ \\
\hline Diabetes, N (\%) & $14(3.3)$ & $8(2.7)$ & $6(4.7)$ & 0.295 \\
\hline Marfan, N (\%) & $15(3.5)$ & $4(1.3)$ & $11(8.5)$ & 0.001 \\
\hline Previously cardiac surgery, N (\%) & & & & 0.865 \\
\hline CAD, N (\%) & $27(6.3)$ & $20(6.7)$ & $7(5.4)$ & 0.616 \\
\hline CKD, N (\%) & $12(2.8)$ & $9(3.0)$ & $3(2.3)$ & 1.000 \\
\hline Cerebral infarction, N (\%) & $8(1.9)$ & $5(1.7)$ & $3(2.3)$ & 0.703 \\
\hline Smoke, N (\%) & $78(18.3)$ & $50(16.8)$ & $28(21.7)$ & 0.226 \\
\hline Alcohol, N (\%) & $49(11.5)$ & $33(11.1)$ & $16(12.4)$ & 0.692 \\
\hline Emergency, N (\%) & $387(90.6)$ & $275(92.3)$ & $112(86.8)$ & 0.075 \\
\hline Tamponade, N (\%) & $83(19.4)$ & $56(18.8)$ & $27(20.9)$ & 0.608 \\
\hline Coronary malperfusion, N (\%) & $149(34.9)$ & $99(33.2)$ & $50(38.8)$ & 0.270 \\
\hline
\end{tabular}

AVR, aortic valve replacement; TEVAR, thoracic endovascular aortic repair; CAD, coronary artery disease; CKD, chronic kidney disease; LVEF, left ventricular ejection fraction.

considered statistically significant.

\section{Results}

\section{Baseline data}

Preoperative demographics are listed in Table 2. The mean age of all patients was $51.1 \pm 12.5$ years, the patients in Root Replacement Group were younger than Root Reconstruction Group. Most patients were male and hypertension with preserved ventricular function. Hypertension patients were more in Root Reconstruction, while more Marfan patients in Root Replacement Group.
Eighty-three patients (56 in Root Reconstruction, 27 in Root Replacement) presenting with cardiac tamponade received emergency surgery directly from emergency room to operative room. And organ malperfusion were checked out based on the description by Pacini in the article (3). Coronary artery malperfusion had presented obviously in our series $(34.9 \%, 149 / 427)$, no different between groups. Cerebral malperfusion $(13.3 \%, 57 / 427)$ and lower extremity malperfusion $(13.6 \%, 58 / 427)$ were also similar.

We have considered more indications for root treatment strategy in our series, which are listed in Table 1. The degree of preoperative AI between two groups was different, $18.5 \%$ patients in Root Reconstruction Group had more 
Table 3 Operative data

\begin{tabular}{|c|c|c|c|c|}
\hline Category & Whole group $(\mathrm{n}=427)$ & Root Reconstruction ( $\mathrm{n}=298$ ) & Root Replacement $(n=129)$ & $P$ valve \\
\hline CPB (min) & $252.5 \pm 78.1$ & $239.2 \pm 71.4$ & $283.4 \pm 84.3$ & $<0.001$ \\
\hline X clamp (min) & $173.6 \pm 68.9$ & $159.1 \pm 70.0$ & $207.0 \pm 53.3$ & $<0.001$ \\
\hline DHCA (min) & $30.7 \pm 9.5$ & $30.0 \pm 9.4$ & $31.8 \pm 8.3$ & 0.034 \\
\hline Ascending aorta & $269(63.0)$ & $164(55.0)$ & $105(81.4)$ & \\
\hline Arch & $57(13.3)$ & $47(15.8)$ & $10(7.8)$ & \\
\hline Descending & $101(23.7)$ & $87(29.2)$ & $14(10.9)$ & \\
\hline Arch surgery, $\mathrm{N}(\%)$ & & & & $<0.001$ \\
\hline Stent & $112(26.2)$ & $97(32.6)$ & $15(11.6)$ & \\
\hline CABG & $29(6.8)$ & $17(5.7)$ & $12(9.3)$ & 0.208 \\
\hline Concomitant, N (\%) & & & & $<0.001$ \\
\hline MVR & $3(0.7)$ & $3(1.0)$ & 0 & \\
\hline MVP & $15(3.5)$ & $2(0.7)$ & $13(10.1)$ & \\
\hline TVP & $7(1.6)$ & $4(1.3)$ & $3(2.3)$ & \\
\hline Re-exploration (number) & $37(8.7)$ & $28(9.4)$ & $9(7.0)$ & 0.460 \\
\hline \multicolumn{5}{|l|}{ Transfusion (units) } \\
\hline
\end{tabular}

CPB, cardiopulmonary bypass; DHCA, deep hypothermia circulation arrest; CABG, coronary artery bypass graft; MVR, mitral valve replacement; MVP, mitral valve repair; TVP, tricuspid valve repair.

than moderate AI $(\geq 2)$ while the rate in Root Replacement Group was $91.5 \%$. The average diameter of aortic sinus was $41.4 \pm 7.3 \mathrm{~mm}$ (Root Reconstruction Group vs. Root Replacement Group, 38.4 45.3 vs. $48.6 \pm 6.2 \mathrm{~mm}, \mathrm{P}<0.001$ ).

We used the assessment system introduced by Dr. Neri to consider the situation of coronary artery ostia dissection involvement as the adding indication (4). Neri A is a first choice to do reconstruction procedure if there is no dilation and AI. Eleven patients with Neri B had Bentall procedure, the other five received root reconstruction. Three patients with Neri C had Bentall procedure, one patient received root reconstruction, and all patients with Neri $\mathrm{C}$ need coronary artery bypass graft the same time (Table 1).

\section{Operative data}

The mean cardiopulmonary bypass, cross-clamp, and DHCA times were $252.5 \pm 78.1,173.6 \pm 68.9,30.7 \pm 9.5$ minutes, respectively. Root Replacement Group presented longer operative time, bypass time and cross-clamp time but similar DHCA time compared with Root Reconstruction Group (Table 3).

\section{Postoperative data and complication}

The 30 days mortality is $7.7 \%$ (33/427) in the whole group, while no difference between two groups. The postoperative complications were similar between two groups, the main 
Table 4 Postoperative data

\begin{tabular}{|c|c|c|c|c|}
\hline Categories & Whole group $(n=427)$ & Root Reconstruction ( $\mathrm{n}=298$ ) & Root Replacement $(n=129)$ & $P$ valve \\
\hline ICU (day) & $7.5 \pm 6.0$ & $7.7 \pm 6.3$ & $7.1 \pm 5.4$ & 0.030 \\
\hline Drainage in 24 hours (mL) & $1,032.9 \pm 823.6$ & $971.2 \pm 769.5$ & $1,120.2 \pm 842.7$ & 0.090 \\
\hline 30 days mortality, $\mathrm{N}(\%)$ & $33(7.7)$ & $24(8.1)$ & $9(7.0)$ & 0.844 \\
\hline
\end{tabular}

ICU, intensive care unit.

Table 5 Complications

\begin{tabular}{|c|c|c|c|c|}
\hline Categories & $\begin{array}{l}\text { Whole group }(\mathrm{n}=427) \text {, } \\
\qquad \mathrm{N}(\%)\end{array}$ & $\begin{array}{l}\text { Root Reconstruction }(\mathrm{n}=298) \\
\qquad \mathrm{N}(\%)\end{array}$ & $\begin{array}{l}\text { Root Replacement }(\mathrm{n}=129), \\
\qquad \mathrm{N}(\%)\end{array}$ & $P$ valve \\
\hline Stroke & $14(3.3)$ & $13(4.4)$ & $1(0.8)$ & 0.074 \\
\hline CRRT & $29(6.8)$ & $22(7.4)$ & $7(5.4)$ & 0.461 \\
\hline Re-intubation & $18(4.2)$ & $14(4.7)$ & $4(3.1)$ & 0.603 \\
\hline Paraplegia & $3(0.7)$ & $3(1.0)$ & 0 & 0.557 \\
\hline Gl bleeding & $2(0.5)$ & $1(0.3)$ & $1(0.8)$ & 0.513 \\
\hline Sepsis & $4(0.9)$ & $4(1.3)$ & 0 & 0.320 \\
\hline
\end{tabular}

CRRT, continuous renal replacement therapy; Gl, gastrointestinal; ICH, intracranial hemorrhage.

complications were stroke (3.3\%), pulmonary failure $(4.7 \%)$ and renal failure with CRRT (6.8\%) (Tables 4,5).

\section{Follow-up}

In the average follow-up time of $34.5 \pm 26.1$ months, survival rates were similar between two groups (Table 6). The rates of freedom from death in 5 years were similar (Figure 2A). Eight cases received second operation at follow-up period (Table S1).

\section{Aortic root events}

There were seven cases meeting the conditions (Table S2). But only one patient with severe $\mathrm{AI}$ and the diameter of aortic root was $50 \mathrm{~mm}$ received redo Bentall procedure one year after the first stage procedure. The other patients have to be observed for further intervention (Figure 2B,C).

\section{Multivariate analysis}

In multivariable logistic regression analysis, pericardial tamponade, $\mathrm{CPB}$ time $>240$ min and organ malperfusion status emerged as independent predictors of operative mortality (Table S3). The methods for aortic root and aortic arch surgery had no significant relationship with mortality.

\section{Discussion}

\section{Mortality and morbidity}

The rate of intervention and survival of ATAAD is increasing through the time. The data from IRAD showed that $90 \%$ patients had received surgical treatment and the mortality had decreased from $25 \%$ in 1995 to $18 \%$ in 2013 (5). The data of China from Sino-RAD revealed that only $52.6 \%$ patients with ATAAD had surgical treatment with a mortality of $5.3 \%(6)$. The patient population may be the key factor determining the difference. Besides the updated surgical methods through the time improved the outcomes, the baseline characteristics, the status after ATAAD onset (such as cerebrovascular accident, cardiac tamponade, malperfusion), and long- 
Table 6 Follow-up survival

\begin{tabular}{|c|c|c|c|c|}
\hline Categories & Whole group $(n=427)$ & $\begin{array}{l}\text { Root Reconstruction } \\
\qquad(\mathrm{n}=298)\end{array}$ & $\begin{array}{l}\text { Root Replacement } \\
\qquad(\mathrm{n}=129)\end{array}$ & $P$ valve \\
\hline Follow-up time & $34.5 \pm 26.1$ & $37.0 \pm 24.0$ & $38.4 \pm 28.0$ & 0.983 \\
\hline Death at follow-up (survival rate), N (\%) & $59(86.2)$ & $41(86.2)$ & $18(86.0)$ & 0.957 \\
\hline Reoperation, N (\%) & $8(1.9)$ & $7(2.3)$ & $1(0.8)$ & 0.445 \\
\hline
\end{tabular}

A

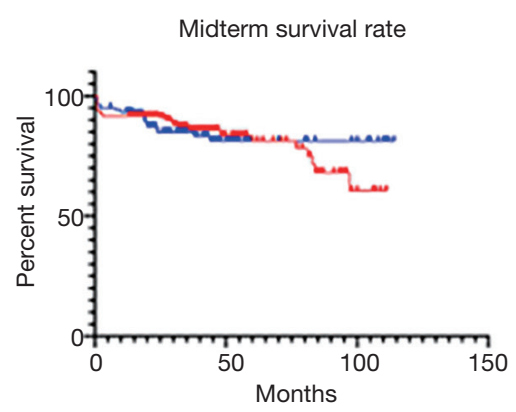

- Root Reconstruction

- Root Replacement
B

Freedom from reoperation

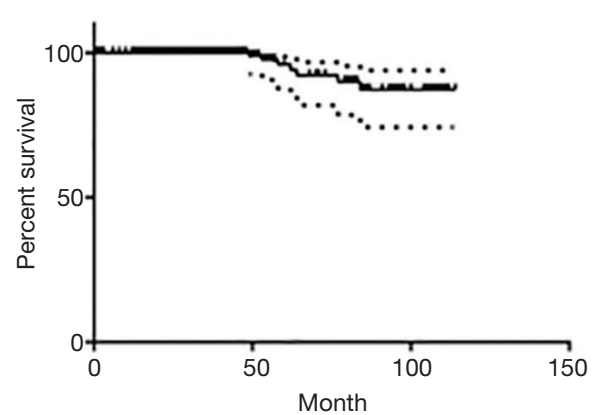

C

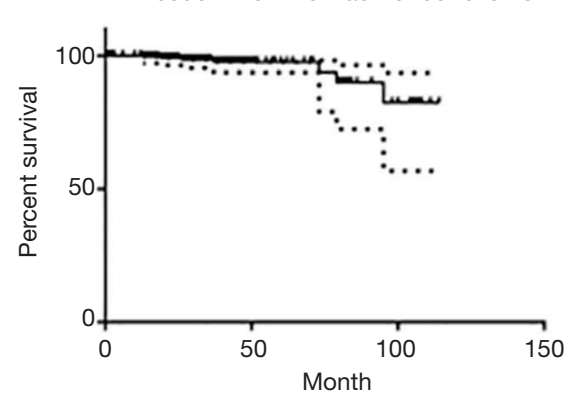

Figure 2 Kaplan-Meier estimate for survival rate and freedom from reoperation and new aortic root events in Root Reconstruction Group. (A) Midterm survival rate was similar between Root Reconstruction Group and Root Replacement Group (P=0.82). (B) Freedom from reoperation during follow-up in the Root Reconstruction Group. (C) Freedom from new aortic root events during follow-up in the Root Reconstruction Group.

time of cardiopulmonary bypass seem as the risk factors of mortality and morbidity (7). The multivariate analysis of our research also revealed the similar results. Neither root treatment nor arch repair affected overall survival in our study and as reported previously (8). One concern about root preservation method is major bleeding from root anastomotic site, which will induce transfusion, postoperative drainage and mortality. The average transfusion volume of red blood cell suspension between this two groups was similar (Root Reconstruction Group vs. Root Replacement Group, $7.4 \pm 4.7$ vs. $7.1 \pm 4.4$ units, $\mathrm{P}=0.440$ ), and the drainage volume in 24 hours was also no different (Root Reconstruction Group vs. Root
Replacement Group, $983.7 \pm 608.6$ vs. $977.9 \pm 601.6 \mathrm{~mL}$, $\mathrm{P}=0.760)$. The courses of bleeding are due to tearing from root anastomotic site when there's no support structure of root and the resistant fragile native intima. After reconstruction by Dacron felts both inside the dissected tissue but also one more layer inside the intima, the stiffness of vascular wall will protect from tearing.

\section{Indications for different root treatment}

As we have mentioned above, the indications for reconstruction or replacement are important before the 
decision made. We considered three main aspects: patients' status, root structure and surgeons' experiences. Patients' status infers the basic characteristics. The results of one research from IRAD revealed compared to Root Replacement Group, the patients in root repair group are older (62.3 years) and the rates of Marfan syndrome (2.5\%) are lower (9). In Marfan and other connective tissue disease population, root replacement should be a more reliable method and the age of connective tissue disease population are much younger than others. We found the same trends but the average age in our whole group are much younger than IRAD.

Marfan syndrome or other connective tissue disease is the independent risk factor influencing the dilation of aortic root. In our series, patients with diagnosed Marfan syndrome received Bentall procedure when the patients' root diameters were $\geq 45 \mathrm{~mm}$ preoperative. We have limited experiences about root repair for Marfan patients and the reoperation for root dilation should still be a question. Recently, more and more surgeon preferred VSRR in the selected patients with Marfan syndrome (10).

The structure of the root contains three aspects: the degree and mechanism of AI, the diameter of aortic root, and the involvement of coronary artery ostia. The mechanisms of AI in ATAAD are discussed and different methods for root treatment based on the mechanism can achieve reliable results (11). True dilation $(\geq 45 \mathrm{~mm})$ represents the dissection is developing from root aneurysm, and Bentall or VSRR procedure is necessary. But the root dilation caused by separation between adventitia and intima is observed in most cases, which can be repaired or reconstructed. VSRR in non-dilated root patients are popular by some experienced surgeons, and the follow-up results showed inferior results with high survival rate and low reoperation rate (12). VSRR seems the complete and good method but the complex manipulation will increase the risk of bleeding and bypass machine related injury, which will restrict widely used.

Another big problem is the involvement of coronary artery ostia, we haven't read similar discussion in the study of root management in TAAD. The three types in Neri classification represent most cases of coronary involvement (4). Neri $C$ need a more radical strategy of CABG, but Neri A and B is debated, Bentall procedure is safe and effective, but the burden of valve replacement are discussed above. The coronary artery ostia in Neri B is partially separated from root, which is hard to fit by our method and the hematoma produced will compress the coronary artery and present myocardial infarction. But in the situation of Neri A, more conservative method will be acceptable, and the 22 cases in our series are all good both in post-operation period and follow-up period.

\section{Root reconstruction in ATAAD}

Direct repair of dissected root is a traditional therapy method for ATAAD. A recent research introduced by Dr. Yang has revealed that direct suture without Teflon felts or biological glue was safe in select patients (13). Only 4\% patients died in their series and it's a huge difference from both IRAD's data and our experiences. As we have discussed above, the method of surgical therapy for aortic root is not the determent factor of mortality. Yang hasn't identified the situation of coronary artery dissection involvement, but the rate of coronary artery involvement in our series is so high that more concern about bleeding and injury of the coronary artery ostium. Yang introduced that using fine 5-0 Prolene sutures and adding the reinforcement around coronary artery ostium, which should be effective but we can't try if there's a more reliable method based on our opinions.

Elimination the dissect root using Teflon felts or glue, and supra-sinus replacement the aorta, the follow-up results revealed that the rate of reintervention of the aortic root because of aortic root dilation or AI was still high. The possible mechanisms are complex, but the structure of aortic root remains the main cause. The native adventitia and intima is separated when the dissection is involving the aortic root, the glue just supports a media to connect the advent and intima, but the diseased aortic root have no assist to support, and the immunological reaction of glue attenuate the constrict characteristic of root, which will induce the root dilation and AI (14). Many surgeons have reconstructed the root using Teflon felts as neomedia and interrupted suture, the reported rate of reoperation of aortic root was lower and acceptable (15). What we have done is using Dacron felts instead of Teflon and adding a more layer inside as neointima and using continuous suture for anastomosis. The results in our center have revealed that the results are similar with other institutes, and the rate of root dilation is as slow as the healthy population. The adding layer have some effects in theory, first the neointima can stand the high flow stress of aorta to avoid the new intimal tear. Secondly, the more layer provides more durable support for aortic root. The difficulties of suturing the four layers can be solved through using Dacron felts instead of Teflon because of the thin structure of Dacron felts. 


\section{Limitations}

The study has some limitations including observational study design, sample size, limited follow-up period, and a single center.

\section{Conclusions}

The rate of survival was similar with other institutes, but the rate of reoperation was lower, even with a much lower rate of root-associated reoperation. It means the reconstruction method for ATAAD in this series, involving the root repair with four-layers structure using Dacron felts, is an acceptable method and worthy to widely promote.

\section{Acknowledgments}

We thank Jason Zhensheng Qu for his editorial assistance.

\section{Footnote}

Conflicts of Interest: The authors have no conflicts of interest to declare.

Ethical Statement: The authors are accountable for all aspects of the work in ensuring that questions related to the accuracy or integrity of any part of the work are appropriately investigated and resolved. The current study was approved by the institutional review board of Nanjing Drum Tower Hospital (No. BL2014004). The requirement to obtain informed consent from the patient was waived.

\section{References}

1. Pan J, Li QG, Zhou Q, et al. Repair of acute type A aortic dissections using open replacement with triple-branched stent grafts. Ann Thorac Surg 2013;96:559-62.

2. Zhou Q, Xue Y, Cao H, et al. Novel arch fenestrated stent graft for acute Stanford Type A aortic dissection with open antegrade implantation. Interact Cardiovasc Thorac Surg 2018;26:369-75.

3. Pacini D, Leone A, Belotti LM, et al. Acute type A aortic dissection: significance of multiorgan malperfusion. Eur J Cardiothorac Surg 2013;43:820-6.

4. Neri E, Toscano T, Papalia U, et al. Proximal aortic dissection with coronary malperfusion: presentation, management, and outcome. J Thorac Cardiovasc Surg 2001;121:552-60.

5. Pape LA, Awais M, Woznicki EM, et al. Presentation,
Diagnosis, and Outcomes of Acute Aortic Dissection: 17Year Trends From the International Registry of Acute Aortic Dissection. J Am Coll Cardiol 2015;66:350-8.

6. Wang W, Duan W, Xue Y, et al. Clinical features of acute aortic dissection from the Registry of Aortic Dissection in China. J Thorac Cardiovasc Surg 2014;148:2995-3000.

7. Hawkins RB, Mehaffey JH, Downs EA, et al. Regional Practice Patterns and Outcomes of Surgery for Acute Type A Aortic Dissection. Ann Thorac Surg 2017;104:1275-81.

8. Fleischman F, Elsayed RS, Cohen RG, et al. Selective Aortic Arch and Root Replacement in Repair of Acute Type A Aortic Dissection. Ann Thorac Surg 2018;105:505-12.

9. Di Eusanio M, Trimarchi S, Peterson MD, et al. Root replacement surgery versus more conservative management during type A acute aortic dissection repair. Ann Thorac Surg 2014;98:2078-84.

10. David TE, David CM, Manlhiot C, et al. Outcomes of Aortic Valve-Sparing Operations in Marfan Syndrome. J Am Coll Cardiol 2015;66:1445-53.

11. Patel PA, Bavaria JE, Ghadimi K, et al. Aortic Regurgitation in Acute Type-A Aortic Dissection: A Clinical Classification for the Perioperative Echocardiographer in the Era of the Functional Aortic Annulus. J Cardiothorac Vasc Anesth 2018;32:586-97.

12. Sievers HH, Richardt D, Diwoky M, et al. Survival and reoperation after valve-sparing root replacement and root repair in acute type A dissection. J Thorac Cardiovasc Surg 2018;156:2076-82.e2.

13. Yang B, Malik A, Waidley V, et al. Short-term outcomes of a simple and effective approach to aortic root and arch repair in acute type A aortic dissection. J Thorac Cardiovasc Surg 2018;155:1360-70.e1.

14. von Oppell UO, Karani Z, Brooks A, et al. Dissected aortic sinuses repaired with gelatin-resorcin-formaldehyde (GRF) glue are not stable on follow up. J Heart Valve Dis 2002;11:249-57.

15. Rylski B, Bavaria JE, Milewski RK, et al. Long-term results of neomedia sinus valsalva repair in 489 patients with type A aortic dissection. Ann Thorac Surg 2014;98:582-8; discussion 588-9.

Cite this article as: Xue Y, Zhou Q, Pan J, Cao H, Fan F, Zhu $\mathrm{X}$, Chong H, Wang D. Root reconstruction for proximal repair in acute type A aortic dissection. J Thorac Dis 2019;11(11):47084716. doi: $10.21037 /$ jtd.2019.10.62 


\section{Supplementary}

Table S1 Reoperation cases

\begin{tabular}{lll}
\hline Case & Operation & Outcome \\
\hline 1 & Thoracoabdominal aortic replacement & Death \\
2 & Debranch and aortic arch reconstruction & Survival \\
3 & Abdominal aorta replacement & Survival \\
4 & Bentall procedure & Survival \\
5 & Abdominal aorta replacement & Survival \\
6 & Abdominal aorta replacement & Survival \\
7 & Abdominal aorta replacement & Survival \\
8 & Thoracoabdominal aortic replacement & Death \\
\hline
\end{tabular}

Table S2 New root events cases

\begin{tabular}{llll}
\hline Case & Group & CT or TTE & Treatment \\
\hline 1 & A & Root diameter $=46 \mathrm{~mm}$; aortic regurgitation $=1.5$ & Follow-up \\
2 & A & Root diameter $=43 \mathrm{~mm}$; aortic regurgitation $=2$ & Follow-up \\
3 & A & Root diameter $=42 \mathrm{~mm}$; aortic regurgitation $=2$ & Follow-up \\
4 & A & Root diameter $=43 \mathrm{~mm}$; aortic regurgitation $=2$ & Follow-up \\
5 & A & Root diameter $=45 \mathrm{~mm}$; aortic regurgitation $=1.5$ & Follow-up \\
6 & B & Root diameter $=47 \mathrm{~mm}$; aortic regurgitation $=1.5$ & Follow-up \\
7 & A & Root diameter $=50 \mathrm{~mm}$; aortic regurgitation $=4$ & Re-do Bentall procedure \\
\hline
\end{tabular}

CT, computed tomography; TTE, transthoracic echo.

Table S3 Multivariate analysis of risk factors related to mortality

\begin{tabular}{lcc}
\hline Categories & P value & HR (95\% Cl) \\
\hline Root surgery & 0.702 & $0.503(0.386-1.897)$ \\
Arch surgery & 0.114 & $0.569(0.408-1.101)$ \\
Pericardial tamponade & $<0.001$ & $3.299(1.884-8.727)$ \\
Coronary malperfusion & 0.004 & $3.015(1.431-6.668)$ \\
Limb malperfusion & 0.033 & $2.238(1.079-5.973)$ \\
CPB $\geq 240$ min & 0.009 & $3.197(1.311-6.381)$ \\
\hline
\end{tabular}

$\mathrm{CPB}$, cardiopulmonary bypass. 